\title{
Analisando o desempenho de bancos de dados orientados a grafos e relacionais sobre discos mecânicos e de estado sólido: uma abordagem comparativa
}

\author{
Priscila Oliveira, Kleber R Stamboni, Jose F Rodrigues-Jr \\ Instituto de Ciências Matemáticas e de Computação (ICMC) \\ Universidade de São Paulo (USP) - São Carlos - SP \\ [priscila2.oliveira,kleber.stamboni,junio] dusp.br
}

\begin{abstract}
Relational databases define the dominant model of the market in data processing; however, recently, other models started to gain attention. In the context of today's world, where more attention is given to relationships between people or things, the graph-oriented model can be very useful. In this work, the performances of the relational database PostgreSQL and of the graph-oriented Neo4j are compared based on their execution time. We also evaluate both the databases with respect to solid-state disk performance. The goal is to elucidate the situations in which each model is more appropriate, providing guidance to users and developers.
\end{abstract}

Resumo. Banco de dados relacionais definem o modelo dominante do mercado em processamento de dados; no entanto, outros modelos começaram a ganhar destaque recentemente. No contexto do mundo atual em que se dá mais atenção às relações entre pessoas ou coisas, o modelo orientado a grafos pode ser de grande utilidade. Neste trabalho, os desempenhos do banco de dados relacional PostgreSQL e do orientado a grafos Neo4j são comparados com base no tempo de execução. Também é verificado o comportamento dos SGBDs tanto para discos mecânicos quanto para os de estado sólido. O objetivo é elucidar a respeito das situações nas quais cada modelo é mais apropriado, provendo orientação a usuários e desenvolvedores.

\section{Introdução}

Sistemas Gerenciadores de Banco de Dados Relacionais (SGBDRs) já existem comercialmente desde a década de 1970, no entanto, ainda são a tecnologia predominante se comparados a outros modelos de bancos de dados [DB-Engines 2017]. Na segunda década dos anos 2.000, no entanto, os modelos concorrentes, muitas vezes denominados NoSQL (Not Only $S Q L$ ), têm se tornado cada vez mais populares. Dado este cenário, uma comparação entre os modelos torna-se desejável, a fim de se entender as limitações e vantagens de cada um de maneira mais esclarecedora.

Assim, neste artigo, é feita uma comparação entre um banco de dados relacional e um banco de dados orientado a grafos; o objetivo é identificar em quais situações cada abordagem é mais vantajosa, e também avaliar se os SGBDRs são mais adequados do que os bancos orientados a grafos. Para isso, PostgreSQL e Neo4j foram as ferramentas selecionadas como representantes da versão relacional e da orientada a grafos para tal comparação. O PostgreSQL foi escolhido por ser o banco de dados open-source mais usado no mercado, e o Neo4j, por ser o banco orientado a grafos também mais usado no 
mercado, assim como reportado no sítio Web DB-Engines (https://dbengines.com/en/ranking).

Para a comparação, foram elaborados testes de desempenho. Os testes são divididos em operações de filtragem de dados, junção, agrupamento e ordenação; e são aplicados sobre diferentes quantidades de dados. Além disso, também foi analisada a influência do armazenamento em disco mecânico (HDD) e do armazenamento em unidade de estado sólido (SSD); os testes foram realizados em ambas as tecnologias para se averiguar o impacto do meio de armazenamento.

Este artigo foi estruturado da seguinte maneira: na Seção 2, são apresentados conceitos relacionados aos modelos estudados; na Seção 3, são mostradas as principais características dos softwares utilizados; na Seção 4, citam-se as configurações do conjunto de software e de hardware, além da contextualização dos dados; na Seção 5, discutem-se as definições de cada teste proposto; por fim, na Seção 6, são apresentadas as conclusões.

\section{Paradigmas de bancos de dados}

A função de um banco de dados é definir uma camada de software que suporta a construção de aplicações oferecendo, de maneira abstrata, acesso rápido, concorrente, e persistente a dados. Os bancos relacionais se diferenciam segundo a maneira como os dados são guardados, modelados e acessados.

\subsection{Bancos de dados relacionais}

Banco de dados relacional, como define o próprio nome, refere-se à tecnologia que armazena dados segundo o modelo relacional, o qual se baseia em relações, compostas por tuplas de elementos ordenados definidos sob domínios que caracterizam atributos. Essas relações são geralmente representadas em linhas, colunas e tabelas. Cada tabela representa uma entidade: alguma 'coisa' ou relacionamento do mundo real - e cada coluna, por sua vez, significa um atributo: alguma característica da 'coisa' em questão [Silberschatz 2006]. Toda tabela possui um atributo especial, chamado de chave, sua função é identificar cada linha e por isso deve ser um valor único para cada uma. Um exemplo pode ser observado na Figura 1.

Bancos relacionais têm como característica marcante a adequação às características ACID (acrônimo para Atomicidade, Consistência, Isolamento e Durabilidade). A linguagem padrão para se comunicar com bancos de dados relacionais é o SQL (Structured Query Language). Ela é dividida em dois conjuntos principais, um para definir os dados (comandos CREATE, ALTER e DROP), e outro para manipulá-los (comandos INSERT, UPDATE, SELECT e DELETE). Como já mencionado, este trabalho usa o banco de dados relacional PostgreSQL, detalhado na Seção 3.1.

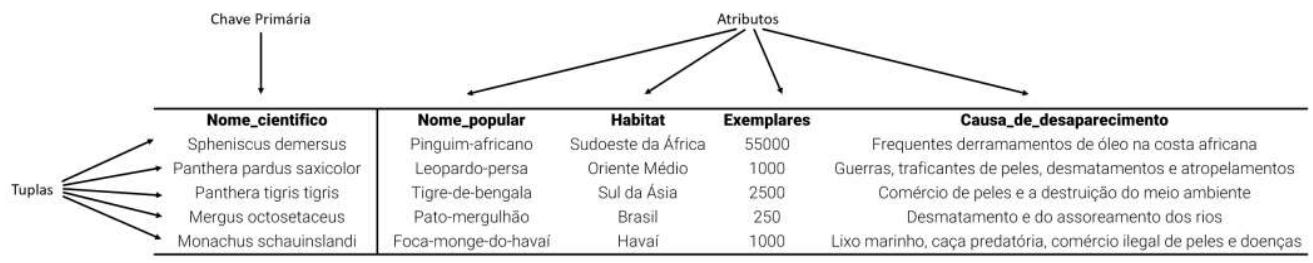

Figura 1. Representação da entidade "Animais_em_extincao" (com informações do sítio Web http://super.abril.com.br/galeria/conheca-20-animais-que-corremrisco-de-extincao). 


\subsection{Bancos de dados orientados a grafos}

Um banco de dados orientado a grafos representa os dados de acordo com a Teoria de Grafos. Um grafo é um modelo matemático capaz de representar relações entre elementos sendo formado por dois conjuntos: um de vértices e um de arestas. Um vértice representa uma entidade - podendo ser do mundo real ou não, já uma aresta representa a conexão entre dois vértices e pode possuir um sentido, uma orientação e, se necessário, dados sobre esse relacionamento [Sato 2014].

A principal motivação em seu uso refere-se a contextos em que a produção de dados define grafos naturalmente, como o exemplo apresentado na Figura 2.

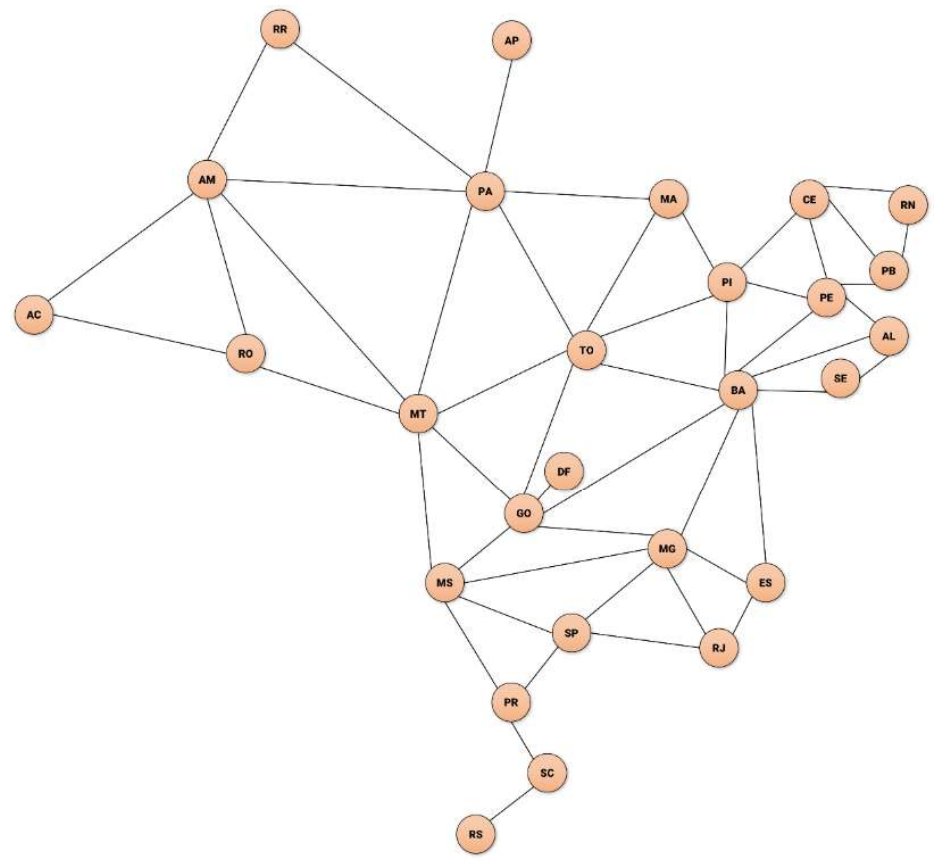

Figura 2. Representação do grafo dos estados brasileiros que fazem fronteira entre si [Feofiloff et al. 2011].

Este trabalho, especificamente, usa o banco de dados orientado a grafos $\mathrm{Neo} 4 \mathrm{j}-$ detalhado na Seção 3.2.

\section{Bancos de dados considerados}

A seguir, as ferramentas usadas nos experimentos são apresentadas.

\subsection{PostgreSQL}

O PostgreSQL é um Sistema Gerenciador de Banco de Dados Objeto-Relacional (SGBDOR) de código aberto. Ele usa a linguagem de consulta SQL, é compatível com ACID e oferece suporte completo para chaves estrangeiras, junções (JOINs), visões, gatilhos, e procedimentos armazenados, entre outros recursos [Comunidade Brasileira de PostgreSQL 2017].

\subsection{Neo4j}

O Neo4j é um banco de dados NoSQL orientado a grafos de código aberto. Ele oferece transações totalmente ACID [Neo4j 2017] — algo incomum para NoSQLs, os quais geralmente proveem consistência BASE (Basically Available, Soft state, Eventual 
consistency), a assim denominada consistência eventual. A linguagem do Neo4j é a Cypher. Ela é uma linguagem declarativa, inspirada em SQL e capaz de definir consultas e dados organizados segundo o modelo de grafos [Sato 2014].

Neste banco de dados, as informações são armazenadas em grafos por meio de nós, e relacionamentos entre eles (ou arestas), ambos podem possuir propriedades que representam suas características.

\subsection{Comparação sintática entre SQL e Cypher}

Uma comparação sintática das cláusulas mais relevantes da linguagem SQL e da linguagem Cypher pode ser visualizada na Tabela 1. A partir dela, notam-se determinadas semelhanças entre as linguagens, também pode-se notar que em alguns casos Cypher é mais conciso.

Tabela 1. Comparação sintática entre SQL e Cypher.

\begin{tabular}{|c|c|c|}
\hline Cláusula & SQL & Cypher \\
\hline Seleção & SELECT * FROM usuario; & $\begin{array}{l}\text { MATCH (u: usuario) RETURN } \\
\text { u; }\end{array}$ \\
\hline Filtragem & $\begin{array}{l}\text { SELECT * FROM usuario WHERE } \\
\text { username LIKE } \\
\text { 'alvstricklan8196'; }\end{array}$ & $\begin{array}{l}\text { MATCH (u: usuario) WHERE } \\
\text { u.username = } \\
\text { 'alvstricklan8196' RETURN } \\
\text { u; }\end{array}$ \\
\hline Junção & $\begin{array}{l}\text { SELECT } \star \text { FROM usuario u } \\
\text { JOIN post p ON u.username = } \\
\text { p.usuario; }\end{array}$ & $\begin{array}{l}\text { MATCH } c=(\text { u: usuario })- \\
{[: \text { PUBLICA }]->(p: \text { post })} \\
\text { RETURN } c ;\end{array}$ \\
\hline Agrupamento & $\begin{array}{l}\text { SELECT u.username, count }(*) \\
\text { FROM usuario u JOIN post p } \\
\text { ON u.username }=\mathrm{p} \text {.usuario } \\
\text { GROUP BY u.username; }\end{array}$ & $\begin{array}{l}\text { MATCH (u: usuario)- } \\
\text { [:PUBLICA]->(p:post) } \\
\text { RETURN u.username, count } \\
\text { (u); }\end{array}$ \\
\hline Ordenação & $\begin{array}{l}\text { SELECT * FROM usuario u } \\
\text { ORDER BY u.nome DESC; }\end{array}$ & $\begin{array}{l}\text { MATCH (u: usuario) } \\
\text { u ORDEUR BY u.nome DESC; }\end{array}$ \\
\hline
\end{tabular}

\section{Ambiente de testes}

A seguir, são mostrados alguns detalhes sobre as características da base de dados usada nos experimentos, além do hardware e software.

\subsection{O hardware}

Os testes foram executados em um computador portátil HP Pavillion 14 V066BR com processador Intel Core i7-4510U de $2.0 \mathrm{GHz}$, memória RAM de 8 GB modelo DDR3L SDRAM a $1600 \mathrm{MHz}$, unidade de disco rígido de $1 \mathrm{~TB}$ a $5400 \mathrm{RPM}$ e unidade de estado sólido Kingston SSDNow V Series com capacidade de 128 GB.

\subsection{O software}

O sistema operacional usado foi o Windows 10 Home Single Language. As versões dos gerenciadores foram PostgreSQL 9.6 e Neo4j Community Edition 3.0.6. Todas as bases de dados foram instaladas em um único computador e as configurações dos softwares foram mantidas como padrão. 


\subsection{Os dados}

O conjunto de dados empregado nos experimentos descreve uma rede social; seu esquema está ilustrado na Figura 3. Ele foi parcialmente obtido usando-se um gerador de dados online [Free Data Generator 2014] e a outra parte foi gerada usando-se um script implementado em Python para criar relacionamentos e completar a base.

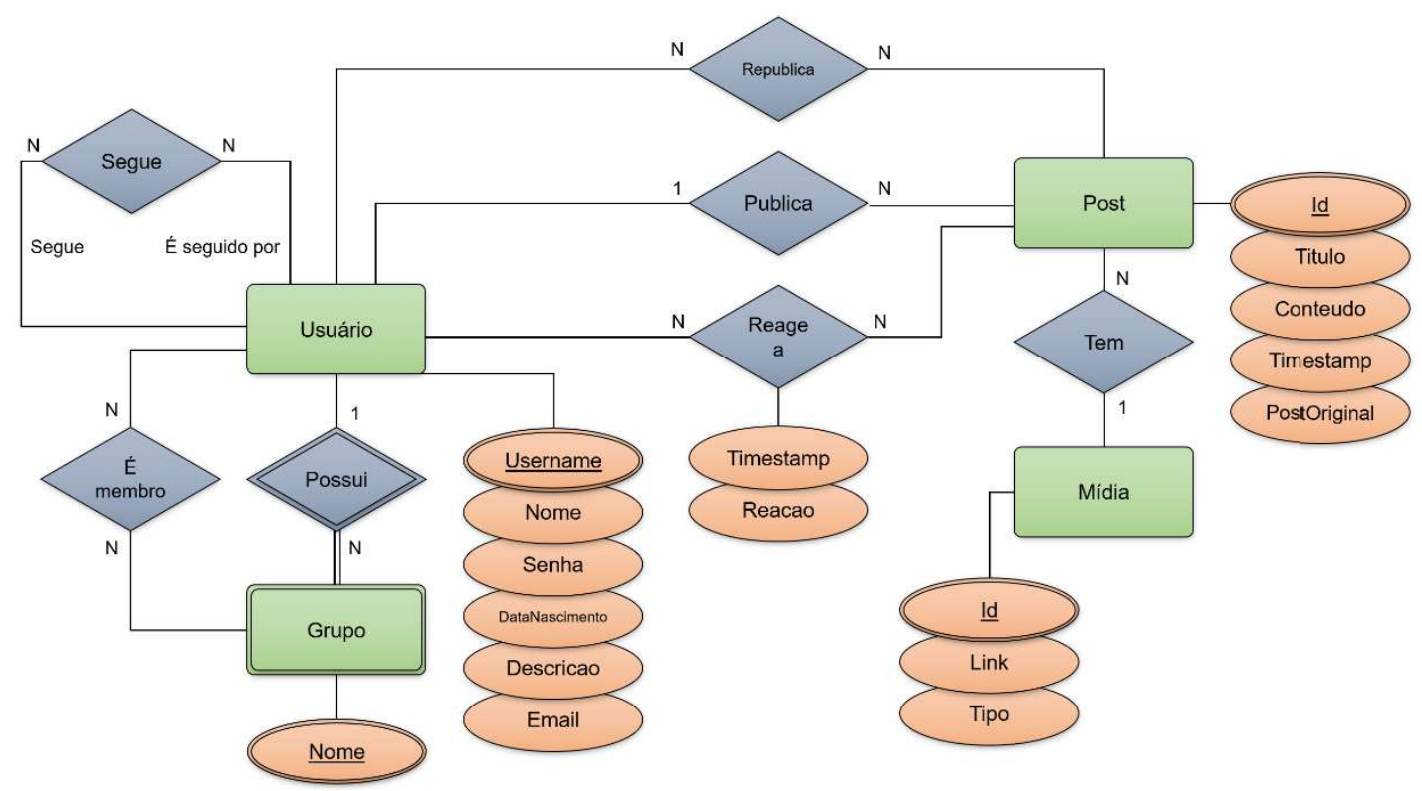

Figura 3. Esquema da base de dados usada nos experimentos.

Os experimentos visam comparar os desempenhos de PostgreSQL e Neo4j usando uma base de dados equivalente em cada uma das ferramentas; ou seja, os dados foram gerados sistematicamente e possuem os mesmos valores em cada banco de dados.

\section{Experimentos}

Os experimentos foram projetados para comparar os dois tipos de gerenciadores de bases de dados considerando a abrangência de operações esperada por softwares desta natureza. Foram consideradas: operações de entrada e saída, filtragem, junção, agrupamento e ordenação. Nesta seção são apresentados detalhes sobre os comandos usados na experimentação; na seção seguinte são exibidos os resultados em termos de espaço de armazenamento e tempo de execução. Foram atribuídos códigos para cada operação a fim de facilitar a identificação de cada uma. A Tabela 2 sumariza os testes realizados e seus respectivos códigos.

Como será detalhado na próxima seção, os experimentos consideraram conjuntos de dados com tamanhos crescentes e dispositivos de armazenamento mecânicos e de estado sólido.

\subsection{Inserção, remoção e tamanho}

Denominou-se a primeira operação como IRT1 - veja a Tabela 2; nesta operação foram obtidos os tempos de inserção de registros na entidade Usuário, veja o esquema na Figura 3. Todas as inclusões necessárias foram feitas sequencialmente por meio de um script.

Do mesmo modo, na operação IRT2, foram obtidos os tempos de remoção dos registros de usuários usando um procedimento equivalente. Por fim, na operação IRT3, analisou-se o espaço ocupado em megabytes pelos registros de usuários. 


\subsection{Filtragem}

Quando é necessário encontrar um subconjunto de tuplas que satisfaçam determinados critérios, conectamos cláusulas com operadores conjuntivos (AND) e disjuntivos (OR). O primeiro deles seleciona tuplas que atendam estritamente a todos os critérios de busca. Já o segundo seleciona tuplas que atendam apenas a um (ou porventura mais de um) desses critérios. Os casos analisados para a base estudada são descritos posteriormente.

Na primeira operação, denominada CD1, é realizada uma consulta conjuntiva, na qual buscam-se usuários que tenham determinado nome, descrição com uma palavra especificada, e que estejam em um intervalo de idade.

Na segunda operação, CD2, uma consulta disjuntiva é definida na qual obtém-se usuários com determinado nome ou domínio de e-mail ou tenham mais do que uma idade especificada.

$\mathrm{Na}$ terceira, CD3, é realizada uma consulta mais complexa, combinando operadores conjuntivos e disjuntivos, na qual obtém-se usuários com determinado nome ou que possuam descrição com uma palavra específica ou um domínio de e-mail e tenham mais do que uma idade especificada.

$\mathrm{Na}$ quarta, CD4, avalia-se o uso de índices. Primeiro obtém-se usuários com uma idade estabelecida; em seguida, realiza-se o teste novamente após a geração dos índices. E por fim, em CD5, é verificado o desempenho das ferramentas na criação e exclusão dos índices.

\subsection{Junção}

Nos bancos de dados relacionais, chaves estrangeiras referenciam chaves primárias de outras tabelas. Assim, junções são realizadas com base na chave primária e na chave estrangeira de instâncias de tabelas. Para a computação de junções, é necessária a operação de produto cartesiano entre conjuntos, o que faz com que esta operação seja das mais custosas.

Banco de dados orientados a grafos não realizam a operação de junção porque são usados os relacionamentos entre os nós para percorrer o grafo. E, portanto, esse é um dos pontos que pode gerar maiores diferenças de desempenho.

Para a operação J1, é analisada uma consulta complexa em SQL para se obter os relacionamentos de terceiro grau entre usuários. Por ser uma informação obtida naturalmente com as arestas dos grafos, a sintaxe em Cypher é mais simplificada. $\mathrm{Na}$ operação J2, a finalidade é avaliar o impacto de uma junção com várias entidades em uma mesma consulta. Veja na Tabela 2.

\subsection{Agrupamento}

Para a operação de agrupamento (A), foram calculadas a idade mínima, a máxima, a média, e a soma das idades de todos os usuários registrados na base. 


\subsection{Ordenação}

Já para a operação de ordenação $(\mathrm{O})$, foram selecionadas e organizadas as tuplas com os registros de usuários, de acordo com o nome.

\subsection{Sumário das operações usadas nos experimentos}

A seguir é apresentada a Tabela 2, a qual sumariza as operações usadas nos experimentos da Seção 6. A tabela tem o propósito de oferecer um panorama dos experimentos e também de servir com referência na interpretação dos resultados.

Tabela 2. Tabela resumo das operações usadas nos experimentos.

\begin{tabular}{|c|c|c|}
\hline $\begin{array}{c}\text { Fator } \\
\text { avaliado }\end{array}$ & Código & Comando ilustrativo SQL e Cypher \\
\hline \multirow{2}{*}{$\begin{array}{l}\text { Inserção de } \\
\text { dados }\end{array}$} & \multirow{2}{*}{ IRT1 } & $\begin{array}{l}\text { INSERT INTO usuario (username, senha, email, } \\
\text { nome, idade, descricao) VALUES ('haydking1322', } \\
\text { 'l3Xp48HUA', 'Hayd. KI9373egmail.com', 'Hayden } \\
\text { King', 14, 'Lorem ipsum ultricies parturient } \\
\text { ante, dolor ipsum sagittis curae; non mattis } \\
\text { nibh.'); }\end{array}$ \\
\hline & & $\begin{array}{l}\text { CREATE (heatwalter8057: usuario\{username: } \\
\text { "heatwalter8057", senha: "shQ2H2", email: } \\
\text { "Heat.WALT40220live.com", nome: "Heather } \\
\text { Walter", idade: 37, descricao: "Lorem ipsum } \\
\text { rutrum habitasse habitant nam nullam felis } \\
\text { adipiscing vel quam tempor."\}); }\end{array}$ \\
\hline \multirow{2}{*}{$\begin{array}{c}\text { Remoção de } \\
\text { dados }\end{array}$} & \multirow{2}{*}{ IRT2 } & DELETE FROM usuario; \\
\hline & & MATCH (m:usuario) DELETE m; \\
\hline \multirow{2}{*}{$\begin{array}{l}\text { Consulta } \\
\text { conjuntiva }\end{array}$} & \multirow[b]{2}{*}{ CD1 } & $\begin{array}{c}\text { SELECT * EROM usuario u WHERE u.nome LIKE } \\
\text { 'Sawyer\%' AND u.descricao LIKE '\%platea\%' AND } \\
\text { u.idade BETWEEN } 13 \text { AND 21; }\end{array}$ \\
\hline & & $\begin{array}{c}\text { MATCH (u: usuario) WHERE u.nome }=\sim \text { '.*Sawyer.*' } \\
\text { AND } \\
\text { u.descricao }=\sim \text { '.*platea. *' AND u.idade }>=13 \\
\text { AND u.idade }<=21 \text { RETURN u; }\end{array}$ \\
\hline \multirow[b]{2}{*}{$\begin{array}{l}\text { Consulta } \\
\text { disjuntiva }\end{array}$} & \multirow[b]{2}{*}{$\mathrm{CD} 2$} & 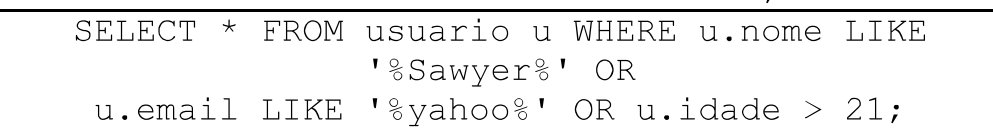 \\
\hline & & $\begin{array}{c}\text { MATCH (u: usuario) WHERE u.nome }=\sim \text { '.*Sawyer.*' } \\
\text { OR } \\
\begin{array}{c}\text { u.email }=\sim \text { '.*yahoo. }{ }^{\prime} \text { OR u.idade }>21 \text { RETURN } \\
u ;\end{array}\end{array}$ \\
\hline \multirow{2}{*}{$\begin{array}{l}\text { Consulta } \\
\text { conjuntiva e } \\
\text { disjuntiva }\end{array}$} & \multirow{2}{*}{ CD3 } & $\begin{array}{l}\text { SELECT * FROM usuario u WHERE u.nome LIKE } \\
\text { '\%Sawyer\%' OR u.descricao LIKE '\%platea\%' OR } \\
\text { u.email LIKE '\%gmail\%' AND u.idade > 21; }\end{array}$ \\
\hline & & $\begin{array}{c}\text { MATCH (u: usuario) WHERE u.nome }=\sim \text { '.*Sawyer.*' } \\
\text { OR } \\
\text { u.descricao }=\sim \text { '.*platea.*' OR u.email }=\sim \\
\text { '.*gmail.*' AND u.idade > } 21 \text { RETURN u; }\end{array}$ \\
\hline \multirow{2}{*}{$\begin{array}{l}\text { Consulta } \\
\text { indexada }\end{array}$} & \multirow{2}{*}{ CD4 } & SELECT $*$ FROM usuario u WHERE u.idade $=21 ;$ \\
\hline & & MATCH (u:usuario\{idade: 21\}) RETURN u; \\
\hline \multirow{2}{*}{$\begin{array}{l}\text { Operações } \\
\text { com índice }\end{array}$} & \multirow{2}{*}{ CD5 } & $\begin{array}{l}\text { CREATE INDEX idade idx ON usuario(idade); } \\
\text { DROP INDEX ON : usuario(idade); }\end{array}$ \\
\hline & & $\begin{array}{l}\text { CREATE INDEX ON : usuario(idade); } \\
\text { DROP INDEX ON : usuario(idade); }\end{array}$ \\
\hline Junção: Tipo 1 & $\mathrm{~J} 1$ & $\begin{array}{c}\text { SELECT b.seguidor, s3. usuarioseguido as } \\
\text { "seguido3" FROM }\end{array}$ \\
\hline
\end{tabular}




\begin{tabular}{|c|c|c|}
\hline & & 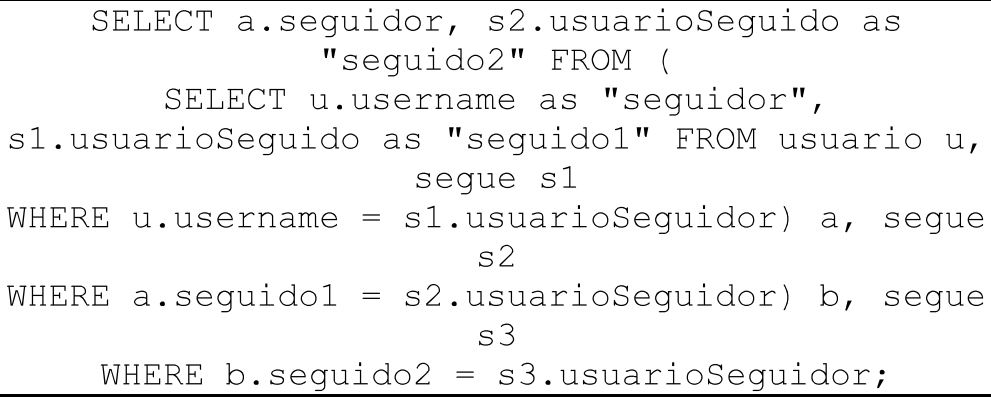 \\
\hline & & $\begin{array}{c}\text { MATCH (a: usuario)-[:SEGUE*3]->(b:usuario) } \\
\text { RETURN a.username, b.username; }\end{array}$ \\
\hline \multirow[t]{2}{*}{ Junção: Tipo 2} & \multirow[t]{2}{*}{$\mathrm{J} 2$} & $\begin{array}{c}\text { SELECT u.username, p.id, g.nome } \\
\text { FROM usuario u JOIN post } \mathrm{p} \text { ON u.username = } \\
\text { p.usuario } \\
\text { JOIN grupo g ON u.username = g.dono; }\end{array}$ \\
\hline & & $\begin{aligned} & \text { MATCH }(g: \text { grupo })<-[: \text { POSSUI] }-(u: \text { usuario })- \\
& \\
& \\
&\text { [:PUBLICA } \mid: \text { REPUBLICA }] \\
&->(p: \text { post }) \text { RETURN u.username, p.id, g.nome; }\end{aligned}$ \\
\hline \multirow{2}{*}{$\begin{array}{l}\text { Agrupamento } \\
\text { de dados }\end{array}$} & \multirow{2}{*}{ A } & $\begin{array}{c}\text { SELECT MIN(idade), MAX(idade), AVG(idade), } \\
\text { SUM(idade) FROM usuario; }\end{array}$ \\
\hline & & $\begin{array}{l}\text { MATCH (m:usuario) RETURN MIN(m.idade), } \\
\text { MAX (m.idade), AVG(m.idade), SUM(m.idade); }\end{array}$ \\
\hline \multirow{2}{*}{$\begin{array}{l}\text { Ordenação de } \\
\text { dados }\end{array}$} & \multirow{2}{*}{$\mathrm{O}$} & SELECT * FROM usuario ORDER BY nome; \\
\hline & & MATCH (m:usuario) RETURN m ORDER BY m.nome; \\
\hline
\end{tabular}

\section{Resultados}

Cada teste foi realizado três vezes, anotando-se o tempo de execução e então calculandose a média aritmética. Para cada teste, os SGBDs foram reiniciados (cold run) para a obtenção de resultados mais precisos. Além disso, o tempo foi comparado considerandose o meio de armazenamento, disco mecânico (HDD) ou unidade de estado sólido (SSD), e usando-se diferentes tamanhos de base de dados - 5 mil, 10 mil, 20 mil, 50 mil e 100 mil tuplas - para avaliar escalabilidade. Os resultados estão organizados de acordo com as categorias e os identificadores expostos na Seção 5.

\subsection{Inserção, remoção e tamanho}

Nas atividades de inserção (IRT1) e remoção (IRT2), e com relação ao tempo e ao espaço ocupado pelos registros (IRT3), o Neo4j mostrou-se com menor eficiência que o PostgreSQL na maioria dos testes realizados.

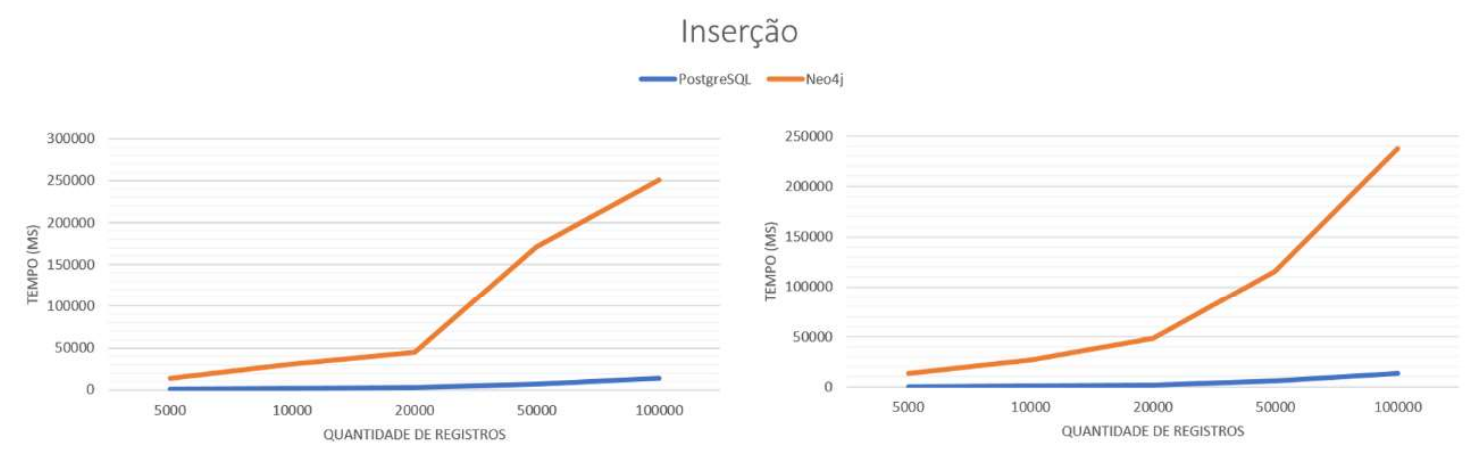

Figura 4. (a) Gráfico comparativo para o tempo da operação IRT1 em HDD; (b) Gráfico comparativo para o tempo da operação IRT1 em SSD. 
$\mathrm{Na}$ operação IRT1, pode-se notar uma perda expressiva de desempenho do Neo4j conforme o número de registros da base de dados cresce, como mostra a Figura 4. O tempo médio de inserção de cada registro para o banco orientado a grafos foi de $2,8 \mathrm{~ms}$ (HDD) e de 2,5 ms (SSD). Para o banco relacional, o tempo médio de inserção foi de 0,2 ms (HDD) e de $0,1 \mathrm{~ms}$ (SSD). O tempo de inserção no SSD foi, em média, 23\% menor para o caso orientado a grafos, e $9 \%$ menor para o relacional.

$\mathrm{Na}$ operação IRT2, o Neo4j tem desempenho praticamente equivalente ao PostgreSQL nas bases menores; no entanto, a performance diminui com o crescimento da base. Pode-se ver esse comportamento na Figura 5. O PostgreSQL terminou em vantagem na maior parte dos casos analisados. Ademais, ambos registraram, em média, eficiência $1 \%$ para o orientado a grafos e $6 \%$ menor em SSD para o caso do relacional.

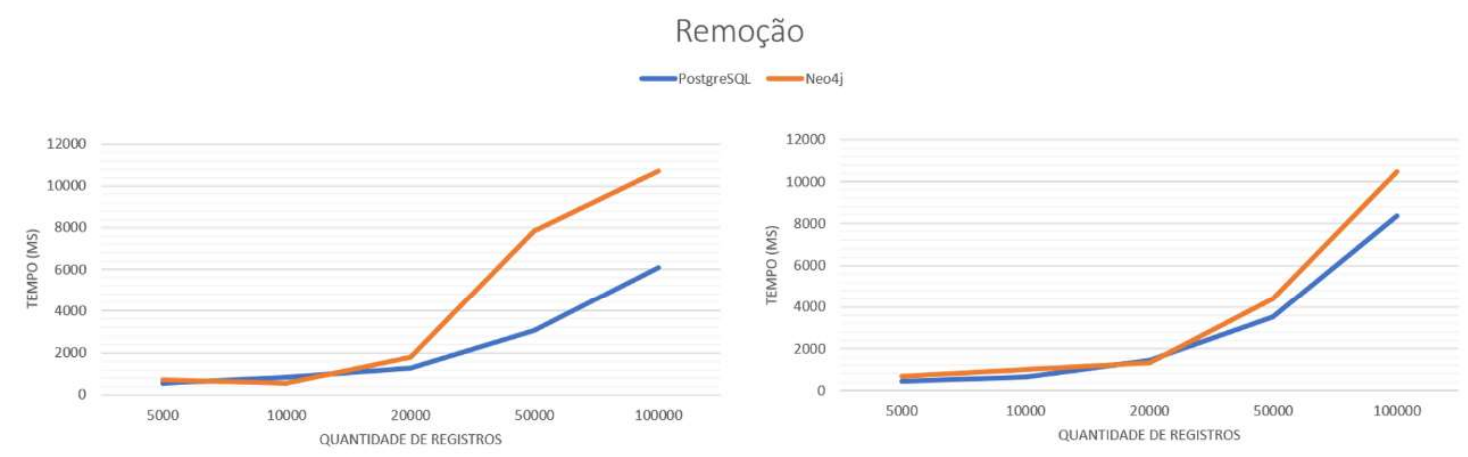

Figura 5. (a) Gráfico comparativo para o tempo da operação IRT2 em HDD (b) Gráfico comparativo para o tempo da operação IRT2 em SSD.

Com relação à análise IRT3, o banco orientado a grafos ocupa menos espaço comparado ao relacional quando são armazenados menos de 20 mil registros; a partir dessa quantidade, o espaço ocupado torna-se maior para o Neo4j. Essa situação é demonstrada na Figura 6. Em média, para todos os casos estudados, o tamanho dos registros em memória secundária do Neo4j é 1,6x maior do que o do PostgreSQL.

\section{Espaço Ocupado}

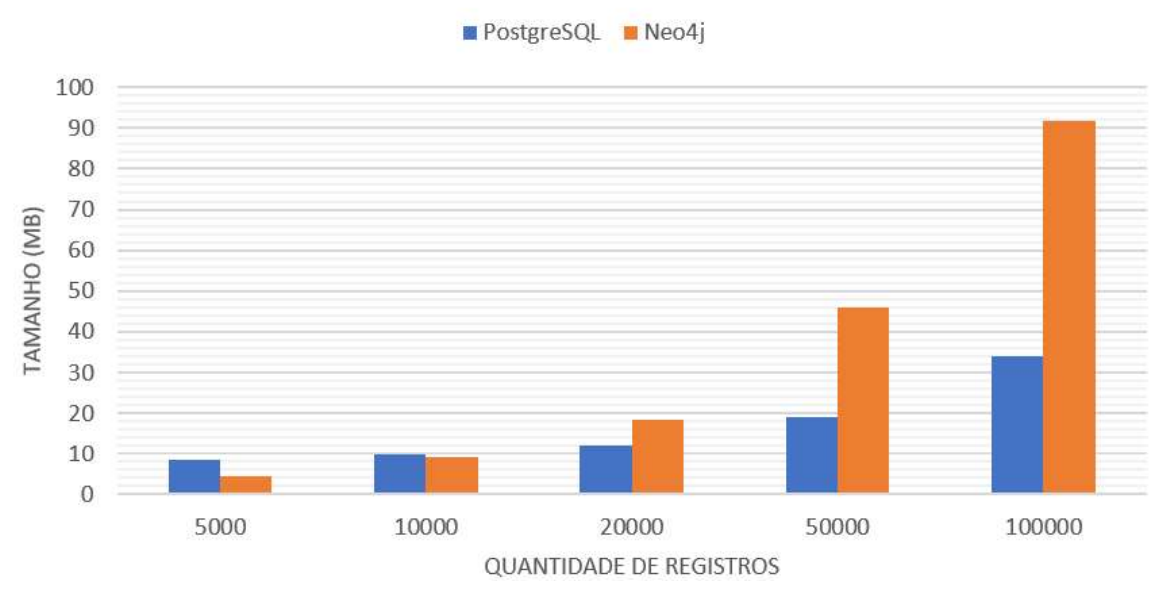

Figura 6. Gráfico comparativo para o espaço ocupado pelos registros armazenados de acordo com o tamanho da base (IRT3).

\subsection{Filtragem}


Na operação CD1, como mostrado na Figura 7, o Neo4j mostrou-se com o melhor desempenho a partir de bases com 20 mil registros para unidade de disco HDD. No entanto, em SSD, o PostgreSQL apresentou melhor desempenho em todos os casos analisados.

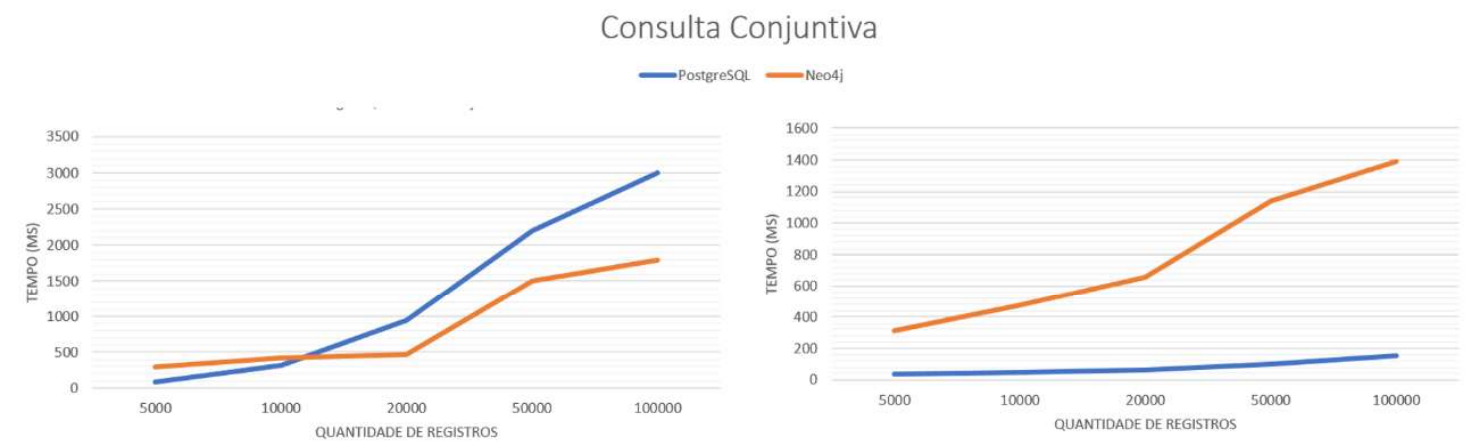

Figura 7. (a) Gráfico comparativo para o tempo da consulta CD1 em HDD; (b) Gráfico comparativo para o tempo da consulta CD1 em SSD.

Para a operação CD2, o banco de dados orientado a grafos obteve o melhor desempenho para todos os casos estudados. No entanto, a eficiência foi $29 \%$ menor, quando comparado com o HDD. Do mesmo modo, o PostgreSQL obteve apenas 3\% a mais de eficiência no disco sólido. Como pode-se ver na Figura 8.

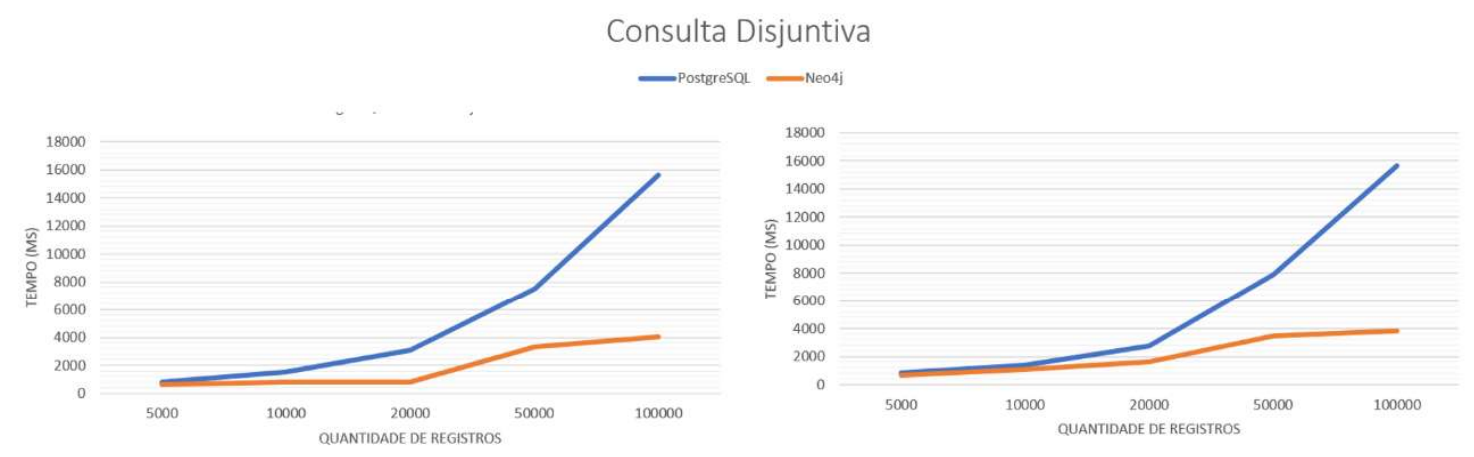

Figura 8. (a) Gráfico comparativo para o tempo da consulta CD2 em HDD; (b) Gráfico comparativo para o tempo da consulta CD2 em SSD.

Em CD3, o Neo4j teve melhor desempenho a partir de 10 mil registros em HDD; em SSD, apenas a partir de 50 mil. Pode-se ver esse comportamento na Figura 9. Além disso, o PostgreSQL executou os testes em um tempo 10\% menor, em média, no SSD; no $\mathrm{Neo} 4 \mathrm{j}$, o desempenho foi oposto, sendo $4 \%$ maior em tempo.

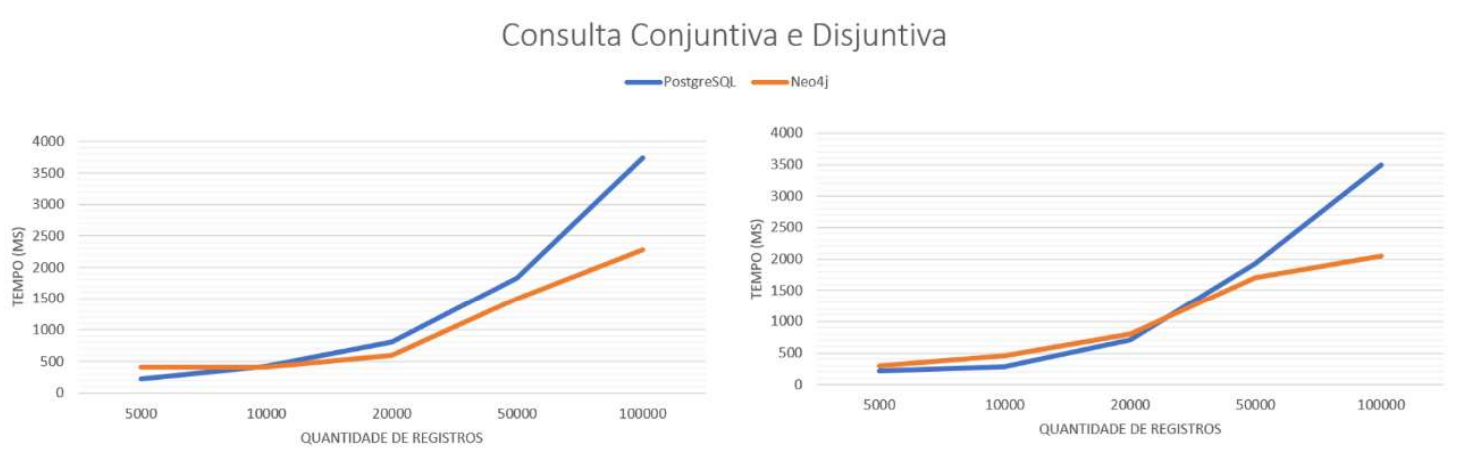

Figura 9. (a) Gráfico comparativo para o tempo da consulta CD3 em HDD; 
(b) Gráfico comparativo para o tempo da consulta CD3 em SSD.

Em CD4, o Neo4j teve melhores resultados para HDD na maioria dos casos; no entanto, em SSD e com índice, o PostgreSQL executou a operação em menos tempo, conforme mostrado na Figura 10. É importante notar que o Neo4j não cria seus índices automaticamente.

\section{Consulta Indexada com 100.000 Registros}

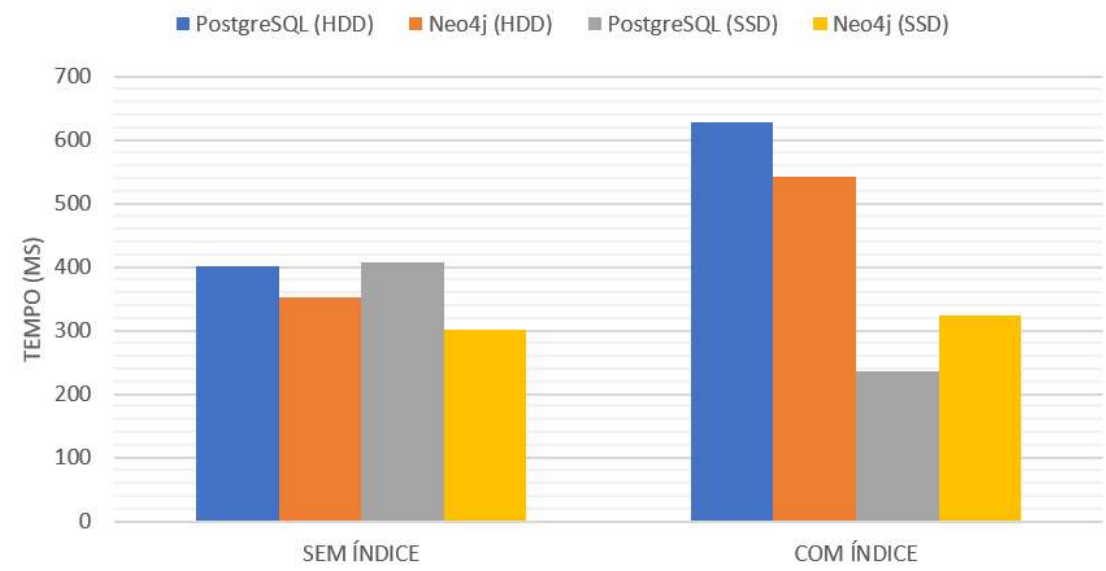

Figura 10. Gráfico comparativo para o tempo da consulta CD4, com e sem índice.

Na Figura 11, são apresentados os tempos de criação e remoção de índices, e como pode-se notar, o banco de dados relacional demora um período bem maior para criá-los, quando comparado com o banco orientado a grafos, tanto em disco sólido como rígido, assim como um período bem menor para excluí-los.

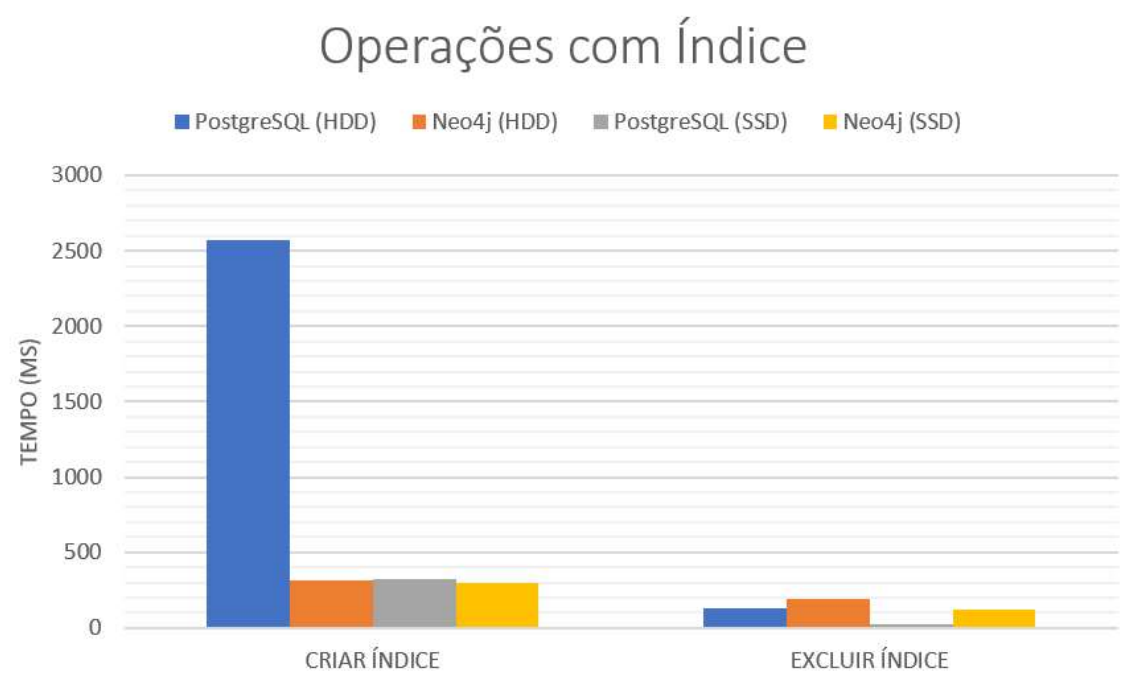

Figura 11. Gráfico comparativo das operações em CD5.

\subsection{Junção}

Nas operações de junção, o Neo4j obteve melhores resultados em SSD, com tempos menores de execução para todos os tamanhos de base estudados em ambos os casos $\mathrm{J} 1$ e $\mathrm{J} 2$. 
Em J1, o banco de dados orientado a grafos, em HDD, obteve melhor tempo para bases até 50 mil registros, porém, para 100 mil, o relacional teve um desempenho levemente superior, como mostra a Figura 12.

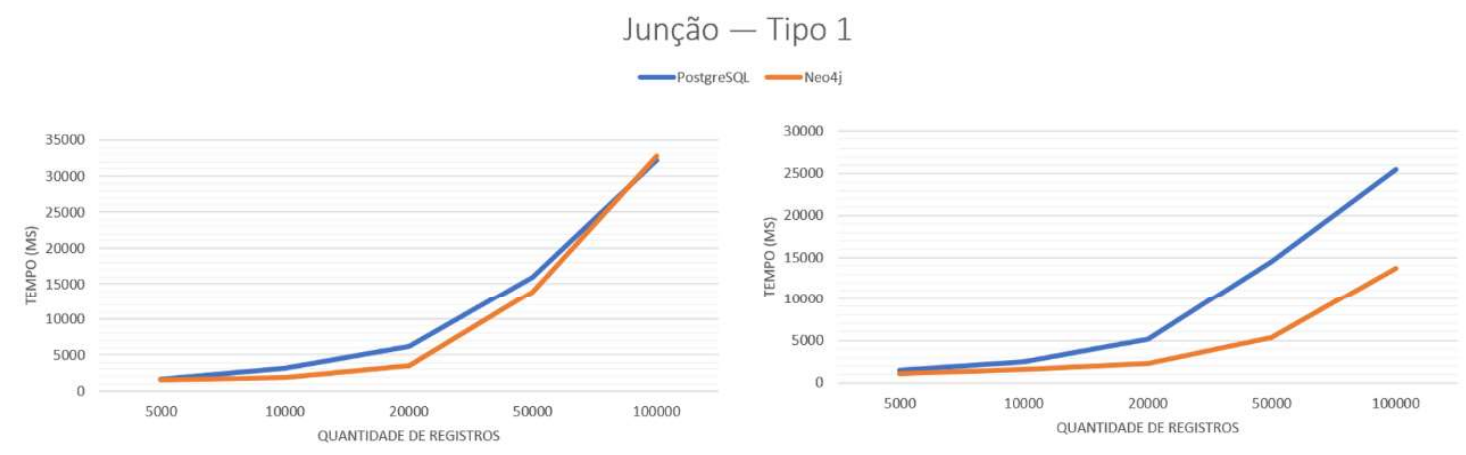

Figura 12. (a) Gráfico comparativo para o tempo da consulta J1 em HDD; (b) Gráfico comparativo para o tempo da consulta J1 em SSD.

Em J2, o Neo4j manteve o desempenho superior para bases até 20 mil registros, todavia, para 50 mil e 100 mil, os menores tempos foram apresentados pelo PostgreSQL (quando em HDD), de acordo com a Figura 13.

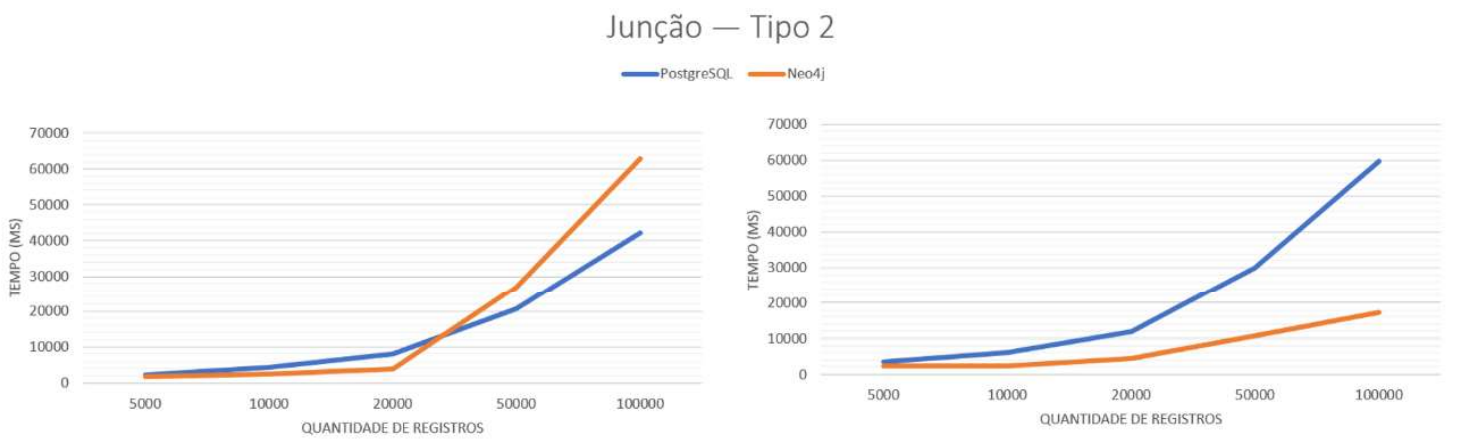

Figura 13. (a) Gráfico comparativo para o tempo da consulta J2 em HDD; (b) Gráfico comparativo para o tempo da consulta J2 em SSD.

\subsection{Agrupamento}

Nesta operação, o Neo4j obteve resultados melhores que o PostgreSQL apenas para bases de 10 mil e 20 mil registros, em disco rígido, como mostra a Figura 14. Além disso, seu desempenho foi inferior no SSD em comparação ao HDD para todos os casos analisados.

Em disco sólido, o banco de dados relacional obteve, em média, ganho de 64\% em eficiência, ao contrário do banco orientado a grafos que perdeu $53 \%$.

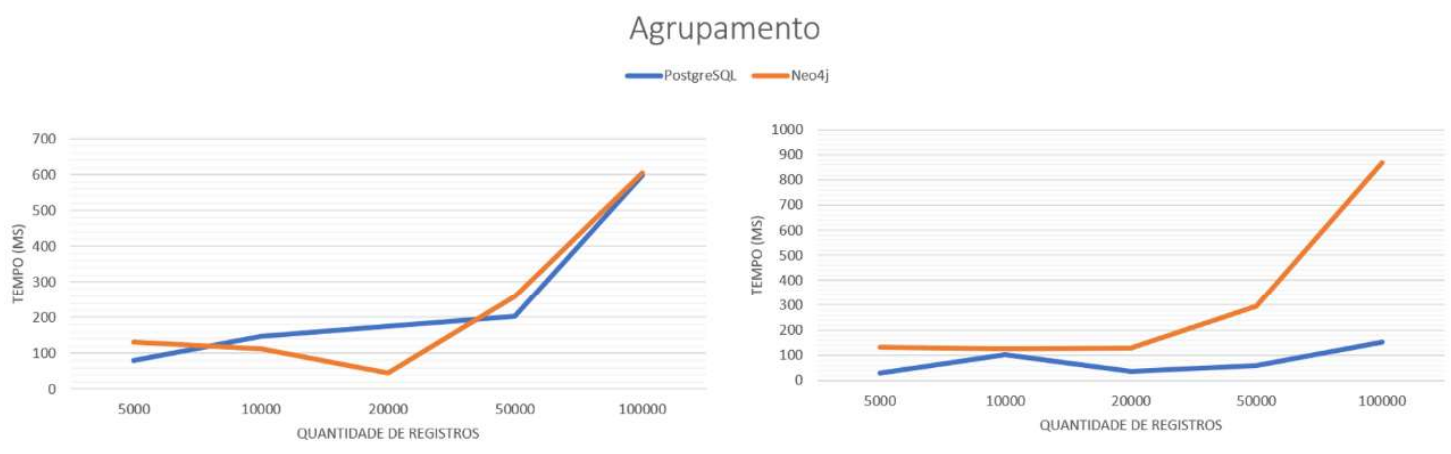


Figura 14. (a) Gráfico comparativo para o tempo da operação A em HDD;

(b) Gráfico comparativo para o tempo da operação A em SSD.

\subsection{Ordenação}

Nesta operação, conforme mostrado na Figura 15, o Neo4j apresentou melhores resultados para todos os casos estudados. O PostgreSQL levou, em média, 3,7x mais tempo para processar a mesma requisição do que o Neo4j em HDD e 2,8x em SSD.

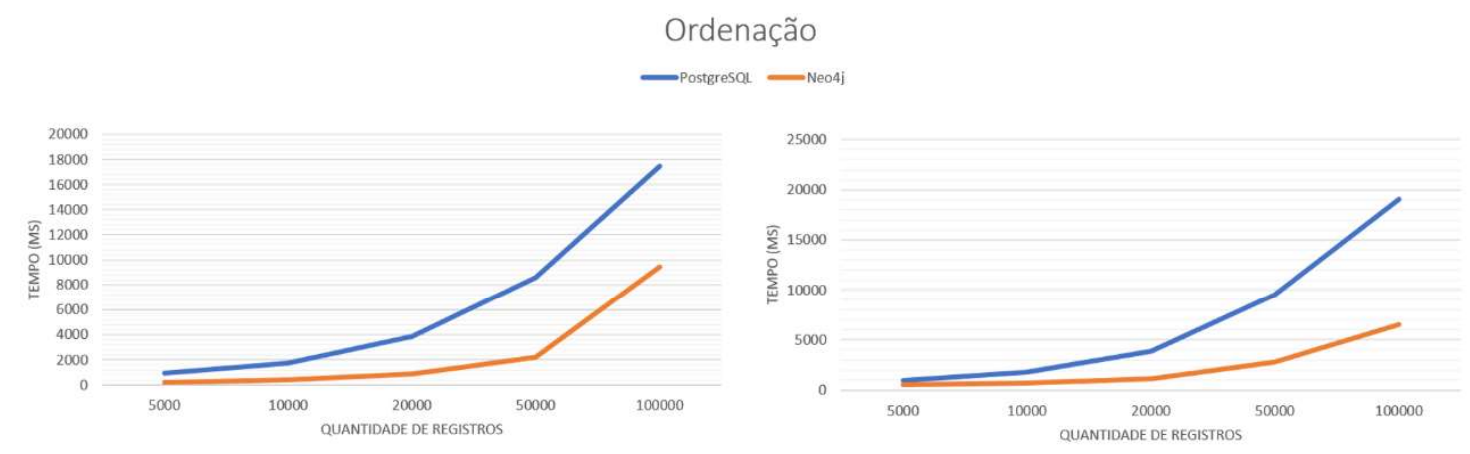

Figura 15. (a) Gráfico comparativo para o tempo da operação $O$ em HDD;

(b) Gráfico comparativo para o tempo da operação 0 em SSD.

\subsection{HDD x SSD}

Por fim, são feitas comparações entre todas as operações realizadas que não envolvem o uso de índice. A Figura 16 mostra os tempos para o disco rígido e para o disco sólido para o PostgreSQL e, na Figura 17, equivalentemente para o Neo4j. É importante notar que as escalas dos gráficos diferem.

\section{PostgreSQL}

- HDD SSD

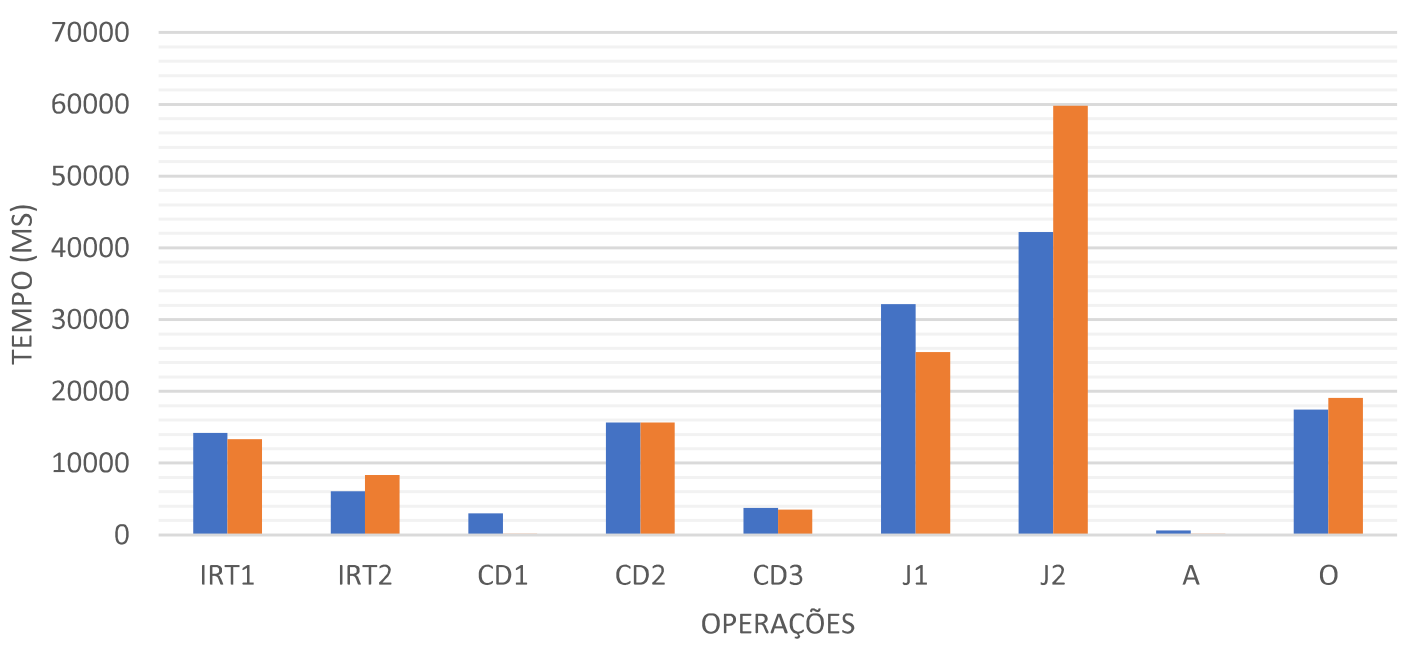

Figura 16. Gráfico comparativo com os tempos das operações em HDD e SSD no PostgreSQL com uma base de 100 mil registros. 


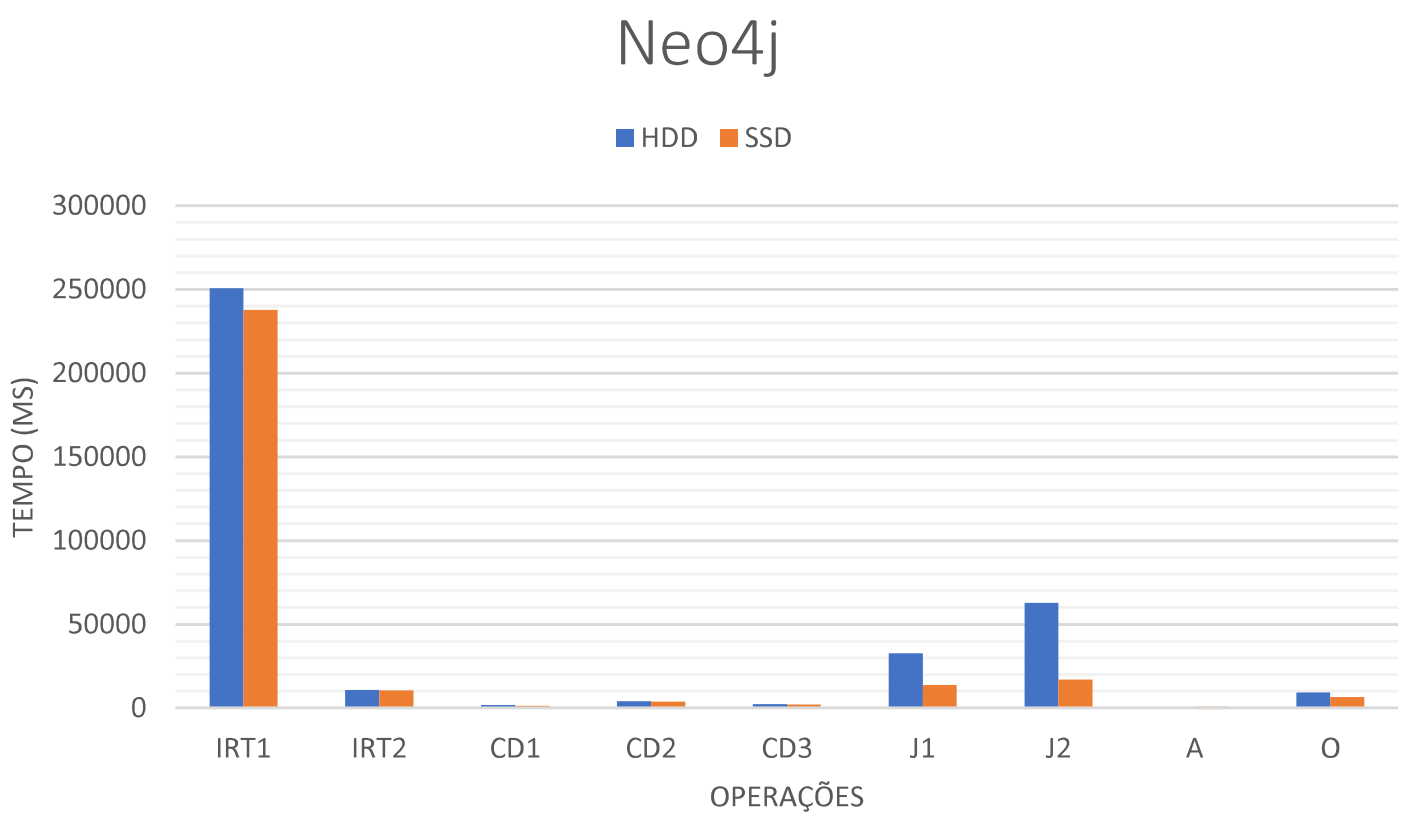

Figura 17. Gráfico comparativo com os tempos das operações em HDD e SSD no Neo4j com uma base de 100 mil registros.

\subsection{Discussão dos resultados}

Em comparação direta de desempenho, foi possível avaliar diversos aspectos do banco de dados relacional PostgreSQL e do banco de dados orientado a grafos Neo4j. Primeiro, ficou claro que o Neo4j requer mais espaço de armazenamento do que o PostgreSQL. Todavia, espaço de armazenamento não é uma questão mandatória atualmente, dado o baixíssimo custo de armazenamento. No que se refere a operações de inserção e remoção, o PostgreSQL se mostrou mais eficiente, o que o torna mais recomendado para sistemas em produção com alta taxa de entrada, como sistemas OLTP (Online Transaction Processing) que suportam operadoras de cartão de crédito, por exemplo. A diferença de desempenho se deve à maneira como o Neo4j organiza os dados em disco, com um arquivo para os nós, um para as propriedades, e um para os relacionamentos (arestas). Cada arquivo requer uma operação adicional em disco durante a escrita. Nota-se que, obviamente, manter arquivos abertos não causa impacto significativo em desempenho; o impacto vem do acesso (disk seek) decorrente do fato de se estar usando dados não contíguos.

A comparação com relação às operações de consulta conjuntivas e disjuntivas não mostraram diferenças de desempenho tão pronunciadas. Dado o fato de que os predicados das consultas se baseiam nas propriedades dos dados, tanto o PostgreSQL quanto o Neo4j resolvem o problema sobre um único arquivo, cujo acesso pode ser otimizado de diferentes maneiras. Com relação ao uso de índices, o Neo4j se mostrou significativamente mais eficiente tanto no uso quanto no gerenciamento.

Nas operações de junção, como se pode esperar, o Neo4j mostrou desempenho superior, especialmente quando usado sobre um disco de estado sólido. Este desempenho é esperado pois o banco orientado a grafos possui um arquivo dedicado ao registro dos relacionamentos entre as entidades do banco, não havendo custo de processamento para realizar a junção cartesiana. Assim, durante a junção, o Neo4j precisa apenas recuperar as entidades envolvidas a partir dos arquivos de nós, uma operação que se torna pronunciadamente mais eficiente em um disco de estado sólido. 
Já com relação à operação de agrupamento, o PostgreSQL mostrou melhor desempenho. Isto ocorre, possivelmente, pois no Neo4j os dados que definem os grupos do agrupamento estão em um arquivo, e os dados de sumarização estão em outro arquivo; ao passo que o PostgreSQL resolve o problema sobre um único arquivo de tuplas.

$\mathrm{Na}$ operação de ordenação, o Neo4j apresentou melhor desempenho, possivelmente porque o arquivo de nós, que é suficiente para a operação de ordenação, é menor do que o arquivo de tuplas do PostgreSQL, o que permite que o algoritmo de ordenação faço melhor uso da memória principal.

Com relação ao uso de SSD e de HDD, ficou claro que o SSD melhora o desempenho dos bancos em praticamente todos os casos. Todavia, o ganho em desempenho não é proporcional ao maior custo dos discos SSD. Com efeito, o GB em SSD ainda é em torno de $700 \%$ mais caro que o GB em um HDD, ao passo que o ganho em desempenho não alcança $200 \%$.

\section{Conclusão}

Os experimentos mostraram que os dois tipos de bancos têm melhor desempenho em diferentes situações. O PostgreSQL se destaca em sistemas de alta taxa de escrita e em operações de agregação para análise de dados. Ao passo que o Neo4j se destaca em operações de consulta e de junção, o que o torna mais adequado a esquemas com muitos relacionamentos e constantes consultas envolvendo junção.

Um dos problemas de se usar o Neo4j vem do fato de que ele não usa SQL; ao passo que a linguagem Cypher não é muito complexa, seu uso por equipes de desenvolvimento pode exigir um tempo de adaptação ou, pior, a reescrita de sistemas já existentes.

Para trabalhos futuros, abrem-se várias possibilidades relacionadas a este assunto, como o estudo com bases de dados ainda maiores para verificar se o desempenho do Neo4j se mantém; a comparação com outros SGBDRs relevantes como Oracle, MySQL e Microsoft SQL Server; e a avaliação do impacto do tamanho da memória principal no tempo das consultas.

\section{Referências}

[Comunidade Brasileira de PostgreSQL 2017] COMUNIDADE BRASILEIRA DE POSTGRESQL. Sobre o PostgreSQL | Comunidade Brasileira de PostgreSQL. Disponível em: $\quad<$ https://www.postgresql.org.br/pages/sobre-o-postgresql.html $>$. Acesso em: 26 dez. 2016.

[DB-Engines 2017] DB-ENGINES. DB-Engines Ranking - popularity ranking of database management systems. Disponível em: $<\underline{\mathrm{http}}: / / \mathrm{db}-$ engines.com/en/ranking >. Acesso em: 26 dez. 2016.

[Feofiloff et al. 2011] FEOFILOFF, P.; KOHAYAKAWA, Y.; WAKABAYASHI, Y. Uma Introdução Sucinta à Teoria dos Grafos. Disponível em:

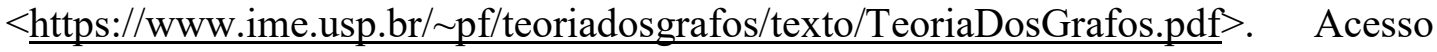
em: 22 maio 2017.

[Free Data Generator 2014] FREE DATA GENERATOR. SQL Data Generator.

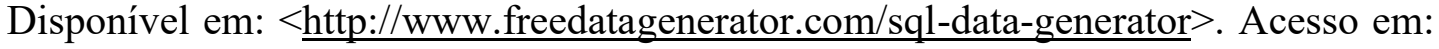
28 jan. 2016. 
[Neo4j 2017] NEO4J. Product At-A-Glance. Disponível em:

$<$ https://info.neo4j.com/rs/neotechnology/images/Product\%20At-A-Glance.pdf $>$. Acesso em: 26 dez. 2016.

[Sato 2014] SATO, P. M. GraphDB Series: o que é um banco de dados de grafos. Disponível em: $<$ https://imasters.com.br/banco-de-dados/graphdb-series-o-que-e-umbanco-de-dados-de-grafos $>$. Acesso em: 22 maio 2017.

[Silberschatz 2006] SILBERSCHATZ, A.; KORTH, H. F.; SUDARSHAN, S. Sistema de Banco de Dados. Tradução de Daniel Vieira. Rio de Janeiro: Elsevier, 2006. p. 11. 